\title{
Flavonoid GL-V9 suppresses invasion and migration of human colorectal cancer cells by inhibiting PI3K/Akt and MMP-2/9 signaling
}

\author{
Ye Gu'1,2,3,4, Jiejie $\mathrm{Yu}^{2}$, Cong Ding1,2,3,4, Yifeng Zhou1,2,3,4, Jiangfeng Yang1,2,3,4, WeiPing Yu ${ }^{5}$, Xiaofeng \\ Zhang ${ }^{1,2,3,4}$, Haitao Huang $1,2,3,4 \bowtie$
}

1. Department of Gastroenterology, Key Laboratory of Clinical Cancer Pharmacology and Toxicology Research of Zhejiang Province, Affiliated Hangzhou First People's Hospital, Zhejiang University School of Medicine, Hangzhou, Zhejiang 310006, P.R.China.

2. Department of Gastroenterology, The Fourth Clinical Medicine College, Zhejiang Chinese Medical University, Hangzhou, Zhejiang 310006, P.R.China.

3. Hangzhou Hospital \& Institute of Digestive Diseases, Hangzhou, Zhejiang 310006, P.R.China.

4. Key Laboratory of Integrated Traditional Chinese and Western Medicine for Biliary and Pancreatic Diseases of Zhejiang Province, Hangzhou, Zhejiang 310006, P.R.China.

5. Department of Pathophysiology, Medical school of Southeast University, Nanjing, Jiangsu 210009, P.R. China.

$\triangle$ Corresponding authors: Xiaofeng Zhang, M.D. Department of Gastroenterology, Key Laboratory of Clinical Cancer Pharmacology and Toxicology Research of Zhejiang Province, Affiliated Hangzhou First People's Hospital, Zhejiang University School of Medicine, Hangzhou, Zhejiang 310006, P.R.China. Tel: +86-0571-56006782; E-mail: zxf837@tom.com; Haitao Huang, M.D. Department of Gastroenterology, Key Laboratory of Clinical Cancer Pharmacology and Toxicology Research of Zhejiang Province, Affiliated Hangzhou First People's Hospital, Zhejiang University School of Medicine, Hangzhou, Zhejiang 310006, P.R.China. Tel: +86-0571-56006782; E-mail: hhtzcn@126.com.

(c) The author(s). This is an open access article distributed under the terms of the Creative Commons Attribution License (https://creativecommons.org/licenses/by/4.0/). See http://ivyspring.com/terms for full terms and conditions.

Received: 2021.01.27; Accepted: 2021.05.22; Published: 2021.06.01

\begin{abstract}
Tumor distant metastasis is the primary cause of death in colorectal cancer (CRC) patients. GL-V9 is a newly synthesized flavonoid derivative with several beneficial biological functions including anti-tumor and anti-inflammation. However, the anti-metastatic effect of GL-V9 and related mechanisms in CRC remains unknown. In this study, the anti-invasive and anti-migratory activities of GL-V9 were investigated in CRC cells. Using MTT assay, cell wound healing assay, and transwell migration assay, we showed that GL-V9 suppressed CRC cell viability, migration, and invasion in a concentration-dependent manner. In addition, the protein expression levels as well as activities of matrix metalloproteinase-2 (MMP-2) and matrix metalloproteinase-9 (MMP-9) were significantly reduced after GL-V9 treatment. Further analysis of the underlying mechanism revealed that GL-V9 inhibited PI3K/Akt signaling pathway upstream of MMP-2 and MMP-9. In conclusion, our study demonstrated that GL-V9 could suppress CRC cell invasion and migration through PI3K/Ak and MMP-2/9 axis. Therefore, GL-V9 might be a potential novel therapeutic agent against CRC metastasis.
\end{abstract}

Key words: GL-V9; colorectal cancer; invasion; matrix metalloproteinases; PI3K/Akt

\section{Introduction}

Colorectal cancer (CRC) is a growing threat to human health which ranks the third commonly diagnosed malignancy and the second leading cause of cancer death worldwide $[1,2]$. Tumor relapse and distant metastasis following conservative surgery are among the major causes of mortality in CRC patients and signify a poor prognosis [3]. Therefore, it is urgent to develop novel anti-metastasis therapeutic agents in order to improve the overall survival of CRC patients.

Cancer invasion and metastasis involve multistep processes including genetic changes and phenotypic alterations of cancer cells [4]. Among these processes the degradation of environmental barriers, such as the extracellular matrix (ECM) and basement membrane is a key event contributing to cancer cell malignancy [5]. Belonging to the family of zinc-dependent endopeptidases, matrix metalloproteinases (MMPs) can collectively degrade nearly all extracellular matrix components and destroy the histological barrier of cancer cell invasion, enabling cancer cells to escape from primary tumor and migrate through basement membrane of blood vessels and connective tissues [6,7]. Among all the MMP 
proteins identified so far, MMP-2 and MMP-9 are considered to be the most crucial members involved in tumor migration and invasion [8]. Overexpression of MMP-2 and MMP-9 are frequently detected in CRC and predict poor prognosis of CRC patients [9-11]. The expression levels of these two proteins are highly correlated with tumor metastasis potential [9-11]. The MAPK/ERK and PI3K/Akt signaling pathways are prominent upstream regulators of MMP-2 and MMP-9. Accumulated evidences have shown that activation of MAPK/ERK and PI3K/Akt promotes CRC invasion and metastasis via up-regulating MMP-2 or MMP-9 expression [12-15]. Therefore, the MAPK/ERK/MMPs or PI3K/Akt/MMPs cascades may serve as promising targets for anti-metastasis drugs [12].

GL-V9 (5-hydroxy-8-methoxy-2-phenyl-7-(4(pyrrolidin-1-yl) butoxy) $4 \mathrm{H}$ - chromen-4-one) is a flavonoid derivative synthesized from the natural product wogonin [16]. The pro-apoptotic, anti-inflammatory, anti-invasive and anti-metastatic effects of GL-V9 have been reported in breast cancer, gastric cancer, and hepatocellular carcinoma [16-19]. However, whether GL-V9 can affect CRC metastasis has remained unclear. In this study, we examined the in vitro effects of GL-V9 on CRC cell viability, invasion and migration. The relevant pathways and underlying molecular mechanisms were further investigated. Our study may provide new evidences for the potential capability of GL-V9 in clinical treatment of CRC.

\section{Materials and methods}

\section{Reagents}

GL-V9 $\left(\mathrm{C}_{24} \mathrm{H}_{27} \mathrm{NO}_{5}\right.$, MW: 409.47, purity > 99\%) was kindly provided by Dr. Bin Di (China Pharmaceutical University, China) and dissolved in dimethyl sulfoxide (DMSO, Sigma-Aldrich, St. Louis, USA) to $0.1 \mathrm{M}$ as a stock sulution and stored at $-20^{\circ} \mathrm{C}$. For cellular experiment, GL-V9 was dissolved in sterilized dimethylsulfoxide (DMSO) and diluted with cell culture medium to different final concentrations. The final DMSO concentration did not exceed $0.1 \% \quad \mathrm{v} / \mathrm{v}$ (volume-to-volume ratio) throughout the study. The PI3K/Akt signaling activator Insulin-like growth factor-1 (IGF-1) and the specific PI3K inhibitor LY294002 was obtained from Beyotime Biotechnology Corporation, Shanghai (Shanghai, China).

Primary antibodies against PI3 Kinase p85, Akt, p-Akt (Ser473), p38 MAPK, p-p38 MAPK (Thr180/ Tyr182), ERK1/2, p-ERK1/2 (Thr202/Tyr204), MMP-2, MMP-9, and $\beta$-actin were obtained from Cell Signaling (Danvers, MA, USA) and were used at a
1:1000 dilution. HRP-conjugated secondary antibodies were obtained from Cell Signaling (Danvers, MA, USA) and were used at a 1:2000 dilution. MTT (3-(4,5)-dimethylthiahiazo(-z-y1)-3,5diphenytetrazoliumromide) was obtained from Sigma-Aldrich (St. Louis, MO, USA).

\section{Cell culture}

Four human CRC cell lines (HCT116, SW480, SW620 and LS174T) and one normal human colon epithelial cell line FHC were purchased from the American Type Culture Collection (Manassas, VA, USA). Cells were cultivated in DMEM medium (Gibco, Carlsbad, CA, USA) supplemented with 10\% heat-inactivated fetal bovine serum (FBS, Gibco, Carlsbad, CA, USA), $100 \mathrm{U} / \mathrm{ml}$ penicillin, and 100 $\mathrm{mg} / \mathrm{l}$ streptomycin. All cells were grown in a stable environment with $5 \% \mathrm{CO}_{2}$ at $37^{\circ} \mathrm{C}$.

\section{Cell viability assay}

The effect of GL-V9 on the viability of CRC cells as well as normal colon epithelial cells was determined by MTT assay. Briefly, cells were seeded at a density of $5 \times 10^{3}$ cells/well onto 96-well microtiter plates containing $100 \mu$ of culture medium and exposed to GL-V9 at different concentrations or equivalent amounts of DMSO as negative control. After treatment for 12, 24 or $48 \mathrm{~h}, 20 \mu \mathrm{l}$ of MTT solution $(5 \mathrm{mg} / \mathrm{ml})$ was added to each well. The plate was incubated at $37{ }^{\circ} \mathrm{C}$ for $4 \mathrm{~h}$ in a humidified atmosphere with $5 \% \mathrm{CO}_{2}$. Then supernatants were removed carefully and $100 \mu \mathrm{D}$ DSO was added to each well. The absorbance was measured at $570 \mathrm{~nm}$ using a microplate autoreader (Bio-Rad, Hercules, CA, USA). The survival rate (\%) was calculated using the formula: Survival Rate $(\%)=\mathrm{OD}_{\text {treated }} / \mathrm{OD}_{\text {control }} \times$ $100 \%$. The inhibition ratio (\%) was calculated using the formula: Inhibition Ratio (\%) = $\left[\left(\mathrm{OD}_{\text {control}}-\mathrm{OD}_{\text {treated }}\right) / \mathrm{OD}_{\text {control }} \times 100 \%\right.$. The IC50 value, which is the concentration that caused $50 \%$ inhibition of cell viability, was calculated using GraphPad Prism v6.0 (GraphPad software, Inc. California, US). The OD values are the average absorbance of three parallel experiments.

\section{Wound healing assay}

Cells were seeded at a density of $1 \times 10^{6}$ cells/ well onto six-well plates and cultured in medium supplemented with 10\% FBS (fetal bovine serum). After the cells grown to $80-90 \%$ confluence, wounds were created in lines across each well using a $10 \mu \mathrm{l}$ sterile pipette tip. The plates were then washed three times with PBS to remove cell debris and replaced with $2 \mathrm{ml}$ of serum-free fresh medium with or without different concentrations of GL-V9. Cells were allowed to grown for $24 \mathrm{~h}$, during which time wound margins 
were photographed and migration was monitored using an inverted microscope. Images were captured using an image-analyzing frame-grabber (LG-3 Scientific Frame Grabber; Scion, Frederick, MD) and analyzed with image analysis software (NIH Image 1.55). The unfilled scratched zones were quantified by measuring the distance between the advancing margins of cells in five randomly selected microscopic fields $(\times 200)$ at each time point. The experiment was performed in triplicates.

\section{Cell invasion assay}

Cell invasion assays were conducted using the transwell assay (Corning Costar, Cambridge, MA, USA). Matrigel was diluted to $5 \mathrm{mg} / \mathrm{ml}$ with DMEM serum-free medium and applied to 8- $\mu \mathrm{m}$-pore polycarbonate membrane filters of the chamber. Cells treated with or without different concentrations of GL-V9 for $24 \mathrm{~h}$ were seeded onto the upper chamber at a density of $5 \times 10^{4}$ cells/well. Then, $300 \mu \mathrm{l}$ of medium with 10\% FBS (fetal bovine serum) was added into the lower chamber. After incubation for 24 $\mathrm{h}$ at $37^{\circ} \mathrm{C}$ in $5 \% \mathrm{CO}_{2}$, cells in the lower chamber were fixed with $1 \%$ paraformaldehyde and stained with haematoxylin after removal of non-invasive cells inside the chamber with cotton swabs. The number of cells migrated through the chamber was counted in five randomly selected fields $(\times 200)$ under a microscope. The experiment was performed in triplicates.

\section{Cell attachment assay}

Cell adhesion assays were performed in 96-well culture plates. The plates were coated with $5 \mathrm{mg} / \mathrm{ml}$ of fibronectin (Sigma, St. Louis, MO, USA) overnight at $4{ }^{\circ} \mathrm{C}$ and blocked with $1 \%$ bovine serum albumin (BSA) in PBS at $37^{\circ} \mathrm{C}$ for $4 \mathrm{~h}$. Cells pre-treated with different concentrations of GL-V9 or without GL-V9 treatment were re-suspended in serum-free DMEM medium at a density of $5 \times 10^{5}$ cells $/ \mathrm{ml}$. Then $100 \mu \mathrm{l}$ of cell suspension was seeded into each well. After incubation at $37{ }^{\circ} \mathrm{C}$ for $1 \mathrm{~h}$, unattached cells were removed by $3 \times$ PBS washes and the adherent cells were quantified by the MTT assay. The experiment was performed in triplicates.

\section{Gelatin zymorgraphy}

The activities of MMP-2 and MMP-9 were detected by gelatin zymography assay as previously described [20]. Cells were exposed to GL-V9 at different concentrations for $24 \mathrm{~h}$, and the supernatant was collected and mixed with loading buffer at a ratio of 3:1 without heating or reduction. The loading volume of each conditioned medium sample was normalized according to the concentration of enzyme detected by BCA assay. The prepared samples were then loaded onto $10 \%$ SDS-PAGE with $0.1 \%$ gelatin before electrophoresis. The gel was washed in $50 \mathrm{mM}$ Tris- $\mathrm{HCl}$ buffer ( $\mathrm{pH} 7.6)$ containing $2.5 \%(\mathrm{v} / \mathrm{v})$ Triton $\mathrm{X}-100$ for $30 \mathrm{~min}$ on a shaker to remove SDS and incubated for $24 \mathrm{~h}$ at $37^{\circ} \mathrm{C}$ in developing buffer (50 $\mathrm{mM}$ Tris- $\mathrm{HCl}$, pH 7.6, $5 \mathrm{mM} \mathrm{CaCl}$, and $1 \mathrm{mM} \mathrm{ZnCl}_{2}$ ). Finally, the gel was stained with $0.1 \%$ Coomassie Brilliant Blue G250 for $1 \mathrm{~h}$ and de-stained in 10\% acetic acid and $10 \%$ methanol.

\section{Western blot}

Cells were lysed on ice in Radio immunoprecipitation assay (RIPA) buffer. Protein concentrations were determined using the Bradford reagent (BioRad, Hercules, CA, USA). Cellular lysates containing equal amounts of total protein were resolved on $12 \%$ SDS polyacrylamide gel and electrotransferred to polyvinylidene fluoride membranes (ImmobilonP; Millipore, Bedford, MA, USA). The membranes were then blocked with $5 \%$ non-fat dry milk in Tris-buffered saline and immunoblotted using the respective primary antibodies overnight at $4{ }^{\circ} \mathrm{C}$. Finally, the appropriate HRP-conjugated secondary antibodies were applied and signals were detected by enhanced chemiluminescence (Pierce, Rockford, IL, USA) following the manufacturer's instructions.

\section{Statistical analysis}

Statistical analyses were performed using SPSS version 20 (SPSS Inc., Chicago, IL, USA). All results shown represent mean \pm S.D. from replicate experiments performed in a parallel manner. Cell migratory rate, invasive ability, and protein expression levels were compared using unpaired student's $t$-test (two-tailed) between two groups and one-way analysis of variance (ANOVA) followed by Tukey's post-hoc test among three or more groups.

\section{Results}

\section{GL-V9 inhibits the viability of CRC cells}

We first investigated the effect of GL-V9 on the viability of CRC cells and normal colon cells. GL-V9 treatment at various concentrations $(0-160 \mu \mathrm{M})$ and durations $(12,24,48$ h) exhibited a dose- and time-dependent inhibitory effect on the viability and proliferation of different CRC cell lines, as determined by the MTT assay (Fig. 1). IC50 values of $24 \mathrm{~h}$ GL-V9-treatment for CRC cells HCT116, SW480, SW620, LS174T and normal cells FHC were $28.08 \pm$ $1.36,44.12 \pm 1.54,36.91 \pm 2.42,32.24 \pm 1.60$, and $81.89 \pm$ $4.26 \mu \mathrm{M}$, respectively (Fig. 1A). Changes in cell viability under $24 \mathrm{~h}$ treatment of GL-V9 were similar to those under $48 \mathrm{~h}$ treatment, but were much greater than those under $12 \mathrm{~h}$ treatment (Fig. 1B). At 
concentrations lower than $20 \mu \mathrm{M}, \mathrm{GL}-\mathrm{V} 9$ has marginal cytotoxic effect on normal colon cells. After $24 \mathrm{~h}$ treatment of GL-V9 at $20 \mu \mathrm{M}$, the inhibitory rates were $(28.50 \pm 2.25) \%,(15.60 \pm 3.15) \%,(24.07 \pm 2.14) \%$, and $(24.50 \pm 3.36) \%$ for HCT116, SW480, SW620 and LS174T cells. However, the inhibitory rate for FHC cells was only $(8.87 \pm 1.21) \%$ (Fig. 1C). Therefore, the concentration range from 0 to $20 \mu \mathrm{M}$ with rare effects on cell viability of normal cells was chosen for the subsequent experiments.

\section{GL-V9 inhibits the migration, invasion, and adhesion of CRC cells}

CRC cell lines HCT116 and SW480 with the highest and lowest sensitivity to GL-V9 treatment were selected for cell migration, invasion, and adhesion assays. To compare the effects of GL-V9 on CRC cells and normal cells, the migration, invasion, and adhesion of normal colon cells FHC with or without GL-V9 treatment were also examined. Treatment of GL-V9 significantly reduced migratory abilities of CRC cells $(p<0.001$; Fig. 2). After migration for $24 \mathrm{~h}$, the extents of wound closure were $(9.50 \pm 2.59) \%$ and $(10.50 \pm 3.30) \%$ for HCT116 and SW480 cells treated with $20 \mu \mathrm{M}$ of GL-V9, but were $(79.67 \pm 5.61) \%$ and $(83.83 \pm 4.49) \%$ for HCT116 and SW480 cells without GL-V9 treatment (Fig. 2). In addition, transwell migration assay indicated that GL-V9 treatment led to remarkable decrease in the invasive capacities of CRC cells $(p<0.001$; Fig. 3A, B). Compared with CRC cells, normal colon cells exhibited much lower migratory property. The extent of wound closure was only $(0.038 \pm 0.02) \%$ for FHC cells without GL-V9 treatment and was $(0.004 \pm$ $0.01) \%$ for FHC cells treated with $20 \mu \mathrm{M}$ of GL-V9 $(p=0.003$; Fig. 2). Similarly, the invasive capacity of FHC cells was much lower than that of CRC cells. The number of FHC cells migrated through the extracellular matrix $(\mathrm{ECM})$ was $11.83 \pm 2.32$ without GL-V9 treatment, but was only $6.5 \pm 2.74$ after treatment of $20 \mu \mathrm{M}$ GL-V9 ( $p=0.005$; Fig. 3A, B).

A key initial step of metastasis is the adhesion of cancer cells to extracellular matrix (ECM) components. Here we also examined the effect of GL-V9 on cell adhesion using the cell attachment assay. GL-V9 treatment significantly reduced CRC cell adhesion as compare with the control group $(p<0.001)$. At a concentration of $20 \mu \mathrm{M}$, the inhibitory rates of cell adhesion were $(56.63 \pm 9.83) \%$ for HCT116 cells and $(48.97 \pm 3.35) \%$ for SW480 cells (Fig. 3C). For FHC cells, treatment of $20 \mu \mathrm{M}$ GL-V9 also significantly reduced cell adhesion $(p=0.005)$, but the inhibitory rates was only (14.02 \pm 5.57$) \%$ (Fig. 3C). Collectively, our results demonstrated that GL-V9 significantly reduced CRC cell migration, invasion, and adhesion in a dose-dependent manner.

\section{GL-V9 suppresses the expression and activities of MMP-2 and MMP-9}

Matrix degrading proteinases play a pivotal role in the degradation of extracellular matrix (ECM), which further contributes to migration and invasion. Therefore, we examined the protein expression levels of MMP-2 and MMP-9 in HCT116 cells exposed to 5, 10, and $20 \mu \mathrm{M}$ GL-V9 for $24 \mathrm{~h}$. Results from western blot analyses showed that treatment of GL-V9 with a concentration $\geq 10 \mu \mathrm{M}$ significantly reduced MMP-2 expression $(p<0.001)$ and MMP-9 expression $(p=0.0033)$ as compared with the control group (Fig. 4A). In addition, gelatin zymography analyses demonstrated that treatment of GL-V9 with a concentration $\geq 10 \mu \mathrm{M}$ resulted in significant decreases in enzyme activities of MMP-2 $(p=0.012)$ and MMP-9 $(p=0.018)$ as compared with the control group $(p<$ 0.05; Fig. 4B).
A

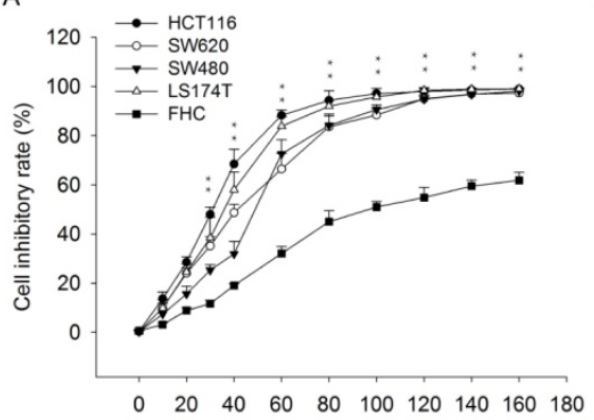

B

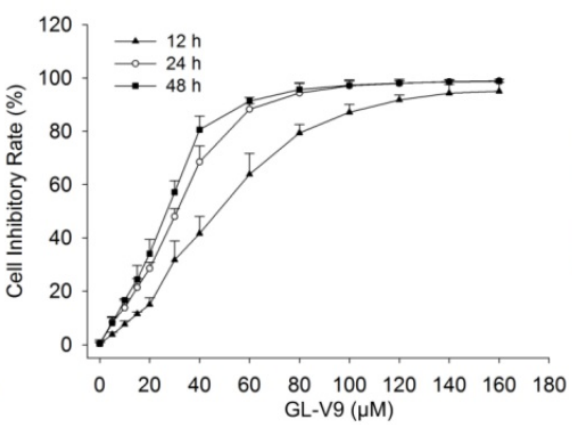

C

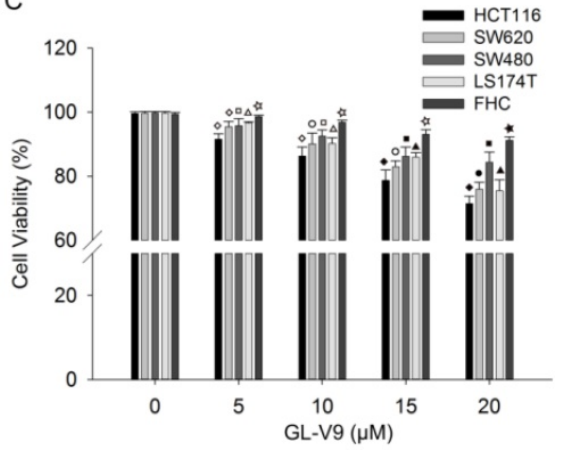

Figure 1. Inhibitory effects of GL-V9 on the viabilities of CRC cells and normal human colon epithelial cells. MTT assays were used to detect cell viability after GL-V9 treatment. (A) CRC cells HCT116, SW620, SW480, LS174T, and normal colon epithelial cells FHC were treated with $0-160 \mu M$ GL-V9 for 24 h. ** p<0.001 for all the examined cell lines. (B) CRC cells HCT116 were treated with 0-160 $\mu$ M GL-V9 for 12, 24, and $48 \mathrm{~h}$. (C) Survival rates of CRC cells HCT1 16, SW620, SW480, LS174T and normal colon epithelial cells FHC treated with $0,5,10,15$, and $20 \mu \mathrm{M} \mathrm{GL}-\mathrm{V} 9$ for $24 \mathrm{~h}$. GL-V9 showed marginal cytotoxic effect on FHC cells even at a concentration of $20 \mu \mathrm{M}$ (inhibition rate $<10 \%$ ). Each error bar represents the mean \pm S.D. of three replicate samples. Symbols on each error bar represent the statistical significance of each cell line. HCT116: $\diamond$ , $p<0.05, \diamond, p<0.001$. SW620: $\circ, p<0.05 ; \bullet, p<0.001$. SW480: $\square, p<0.05 ; \mathbf{m}, p<0.001$. LS174T: $\Delta, p<0.05 ; \Delta, p<0.001$. FHC: $\downarrow$,,$p<0.05 ; \star, p<0.001$. 


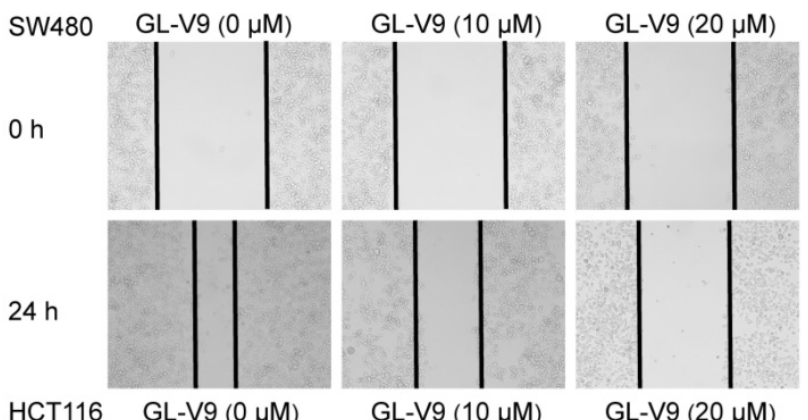

HCT116 GL-V9 $(0 \mu \mathrm{M})$

$\mathrm{Oh}$

$4 \mathrm{~h}$

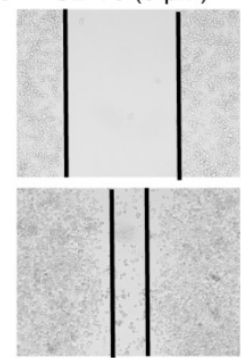

FHC

GL-V9 $(0 \mu \mathrm{M})$

GL-V9 $(10 \mu \mathrm{M})$

GL-V9 $(20 \mu \mathrm{M})$
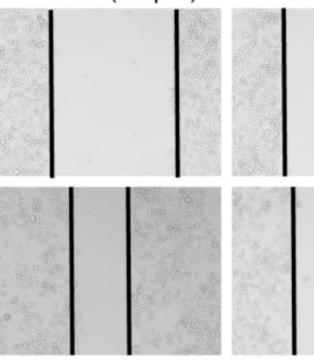

GL-V9 $(10 \mu \mathrm{M})$

GL-V9 $(20 \mu \mathrm{M})$

$0 \mathrm{~h}$
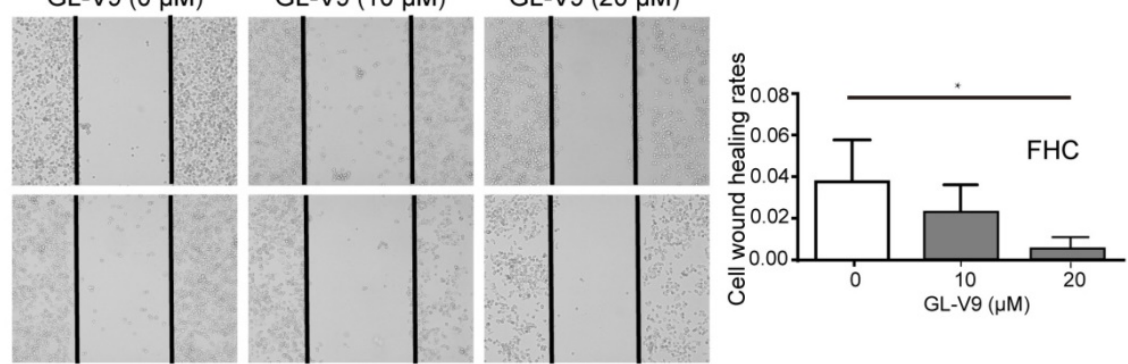

Figure 2. Effect of GL-V9 on cell migration in vitro. CRC cells HCT116 and SW480 treated with $10 \mu \mathrm{M}$ and $20 \mu \mathrm{M}$ GL-V9 for 24 h demonstrated significantly reduced migratory capacities, as indicated by wound healing assay. Normal colon epithelial cells FHC with much lower migration potential also showed reduced migration after GL-V 9 treatment. The representative photographs (A) and quantification (B) are shown. Images were taken at 0 and 24 h. Each error bar represents the mean \pm S.D. of three replicate samples. * $p<0.05$, ** $p<0.001$

\section{GL-V9 suppresses PI3K/Akt signaling}

To explore the possible mechanism underlying the anti-cancer effect of GL-V9, we investigate the correlation between GL-V9 treatment and the activities of PI3K/Akt signaling and MAPK/ERK signaling which are crucial to cell proliferation, migration, and invasion. PI3K expression and Akt phosphorylation was significantly reduced in GL-V9-treated cells, yet the abundance of total Akt did not show evident change (Fig. 5). By contrast, GL-V9 showed little effect on the expression of p38 MAPK and ERK1/2 in total and phosphorylation forms (Fig. 5). These findings suggest that GL-V9 probably inhibits CRC cell migration and invasion by interfering with PI3K/Akt signaling.

\section{Involvement of PI3K/Akt pathway in GL-V9- mediated suppression of MMPs}

Given the regulatory effect of GL-V9 on PI3K/Akt signaling, we sought to examine whether the PI3K/Akt signaling pathway was involved in GL-V9-induced down-regulation of MMP-2 and
MMP-9. CRC cells HCT116 and normal colon cells FHC were exposed to IGF-1, a PI3K/Akt signaling activator. After treatment of $20 \mathrm{ng} / \mathrm{ml}$ of IGF-1 for $2 \mathrm{~h}$, the expression of pAkt, PI3K, MMP-2, and MMP-9 were significantly increased (Fig. 6). However, this effect was reversed when the cells were pre-treated with $20 \mu \mathrm{M}$ GL-V9 for $24 \mathrm{~h}$ followed by IGF-1 treatment (Fig. 6). On the contrary, a specific PI3K inhibitor, LY294002, significantly inhibited Akt phosphorylation and reduced PI3K, MMP-2, and MMP-9 expression. Cells pre-treated with $20 \mu \mathrm{M}$ LY294002 for $24 \mathrm{~h}$ before exposure to IGF-1 for $2 \mathrm{~h}$ showed similar changes in the activity of PI3K/Akt signaling as well as MMP-2/-9 expression with those pre-treated with GL-V9 (Fig. 6). As expected, the expression levels of Akt, pAkt, PI3K, MMP-2, and MMP-9 were lower in normal cells than in CRC cells, yet either IGF-1 treatment or combined treatment of IGF-1+LY294002/GL-V9 led to similar but less evident changes in the expression of these key proteins (Fig. 6). Taken together, the inhibitory effect of GL-V9 on MMP-2 and MMP-9 may result from suppression of the PI3K/Akt signaling pathway. 
A SW480

$24 \mathrm{~h}$

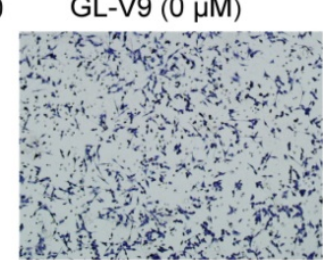

HCT116

GL-V9 $(0 \mu \mathrm{M})$

$24 \mathrm{~h}$

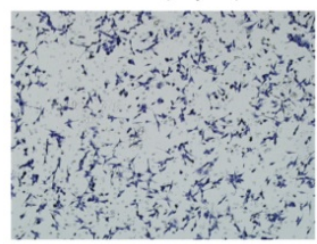

$\mathrm{FHC}$

GL-V9 $(0 \mu \mathrm{M})$

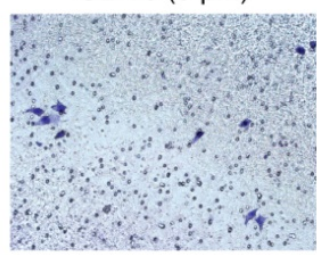

GL-V9 $(10 \mu M)$

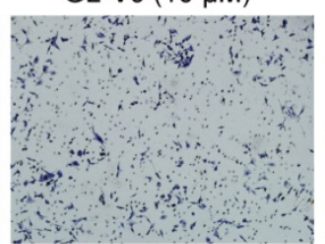

GL-V9 $(10 \mu \mathrm{M})$

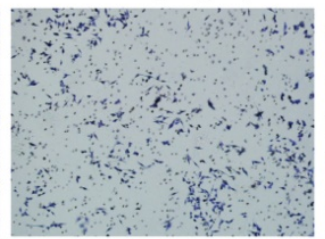

GL-V9 $(10 \mu \mathrm{M})$

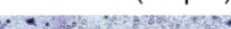

GL-V9 $(20 \mu \mathrm{M})$

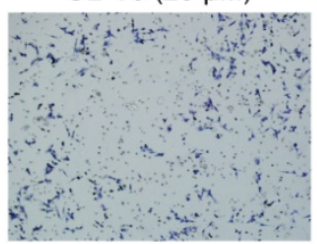

GL-V9 $(20 \mu \mathrm{M})$

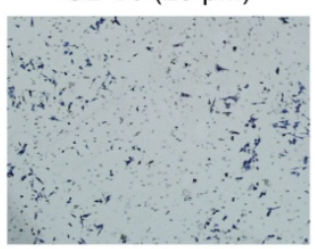

GL-V9 $(20 \mu M)$

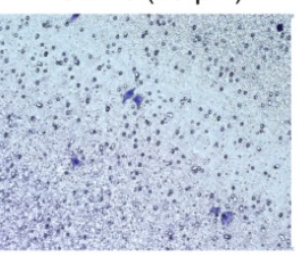

B
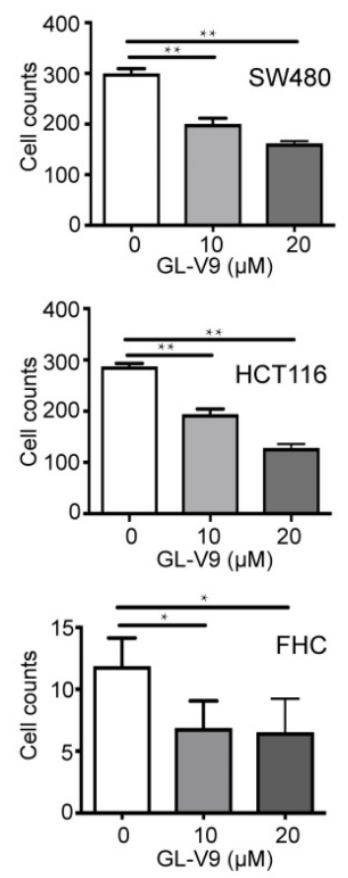

C
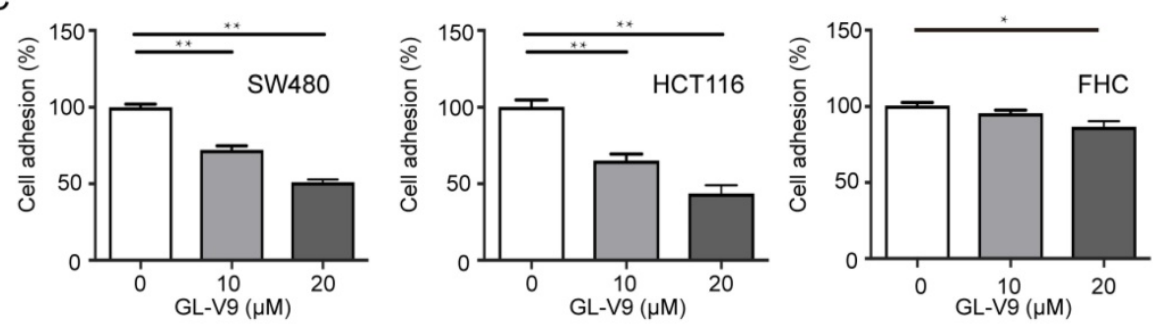

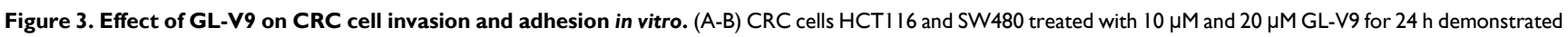
significantly reduced migration and invasion through extracellular matrix as indicated by the transwell migration assay. Normal colon epithelial cells FHC with much lower invasive potential also showed reduced invasion through the extracellular matrix (ECM) after GL-V9 treatment, but with less reduction degree and statistical significance. The number of cells migrated through the ECM after $24 \mathrm{~h}$ was counted in five randomly selected $(\times 200)$ microscopic fields. (C) GL-V9 treatment for $24 \mathrm{~h}$ at a concentration of $20 \mu \mathrm{M}$ significantly reduced cell adhesion for both CRC cells HCTI 16 and SW480 and normal cells FHC, as indicated by cell attachment assay. Each error bar represents the mean \pm S.D. of three replicate samples. $* p<0.05 ; * *, p<0.001$.

A GL-V9 $(\mu \mathrm{M})$ MMP-2

MMP-9

$\beta$-actin

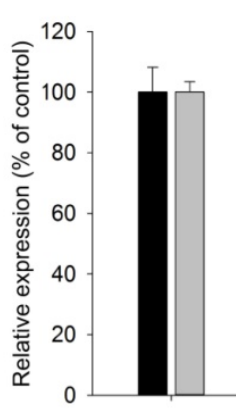

GL-V9 $(\mu \mathrm{M}) \quad 0$

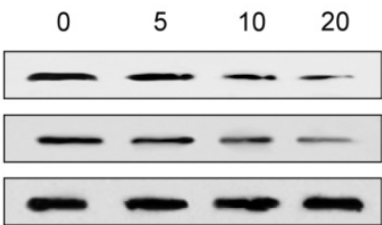

B
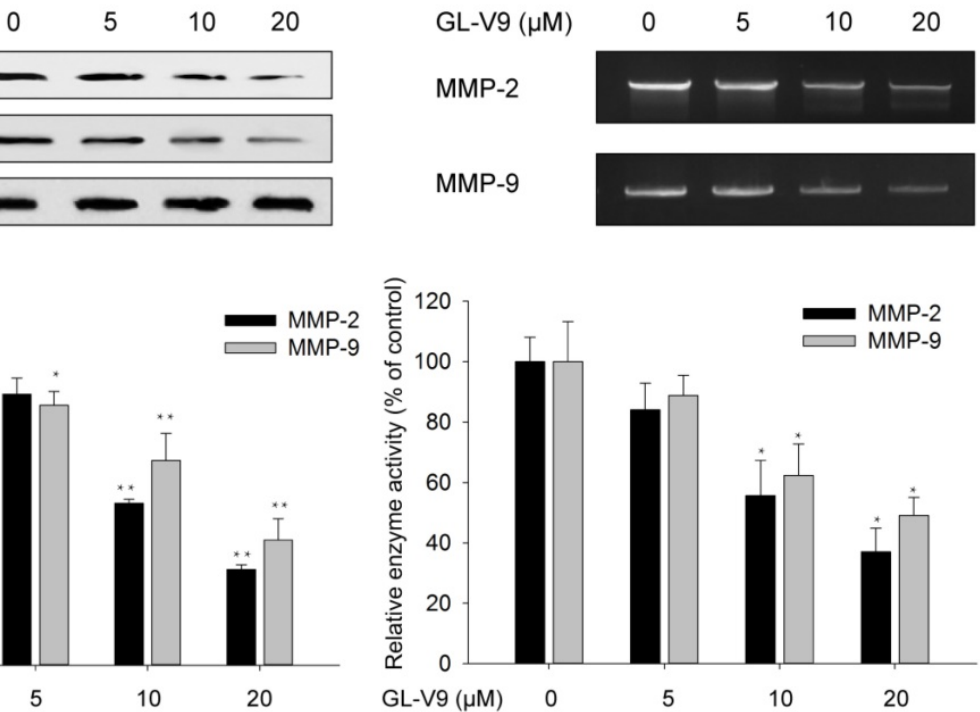

Figure 4. Changes in the expression and activities of MMP-2 and MMP-9 after GL-V9 treatment. (A) Treatment of 5, 10, and $20 \mu M$ GL-V9 for $24 \mathrm{~h}$ in HCT116 cells led to decreased MMP-2 and MMP-9 protein expression. Relative intensities of western blot bands were quantified using the ImageJ software. The expression levels were normalized by $\beta$-actin expression, which was used as internal control. (B) Treatment of 5, 10, and $20 \mu \mathrm{M} \mathrm{GL-V} 9$ for $24 \mathrm{~h}$ in HCT116 cells led to reduced activities of MMP-2 and MMP-9, as indicated by gelatin zymorgraphy. Each error bar represents the mean \pm S.D. of three replicate samples. $* p<0.05, * * p<0.01$. 
A

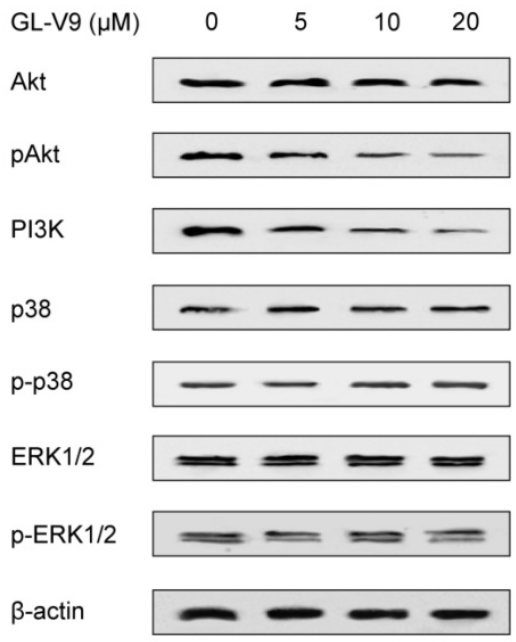

$\mathrm{B}$

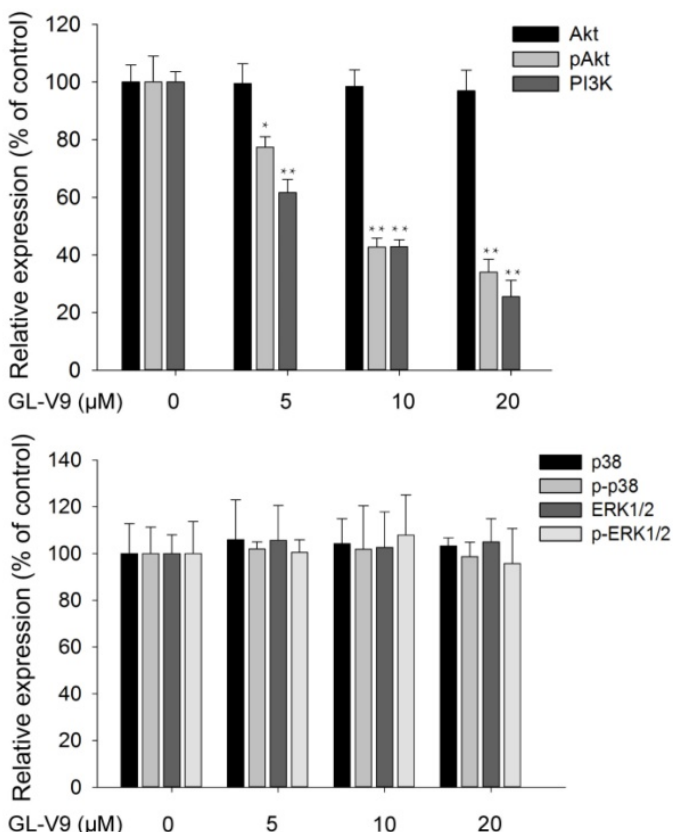

Figure 5. Effects of GL-V9 on the PI3K/Akt and MAPK/ERK signaling pathways. (A) Treatment of 5, 10 , and $20 \mu \mathrm{M}$ GL-V9 for $24 \mathrm{~h}$ in HCT1 16 cells inhibited PI3K/Akt activity in a dose-dependent manner, but did not affect the activity of MAPK/ERK signaling. PI3K expression and phosphorylated form of Akt were significantly reduced in GL-V9-treated cells, but expression of P38 MAPK and ERK1/2 in both the total and phosphorylated forms showed little change after GL-V9 treatment. (B) Quantification of the relative protein expression levels of PI3K, Akt, p-Akt, p38, p-p38, ERK1/2, and p-ERK1/2 in HCT116 cells treated with different concentrations of GL-V9, as compared with the control group. Each error bar represents the mean \pm S.D. of three replicate samples. $* p<0.05, * * p<0.01$.
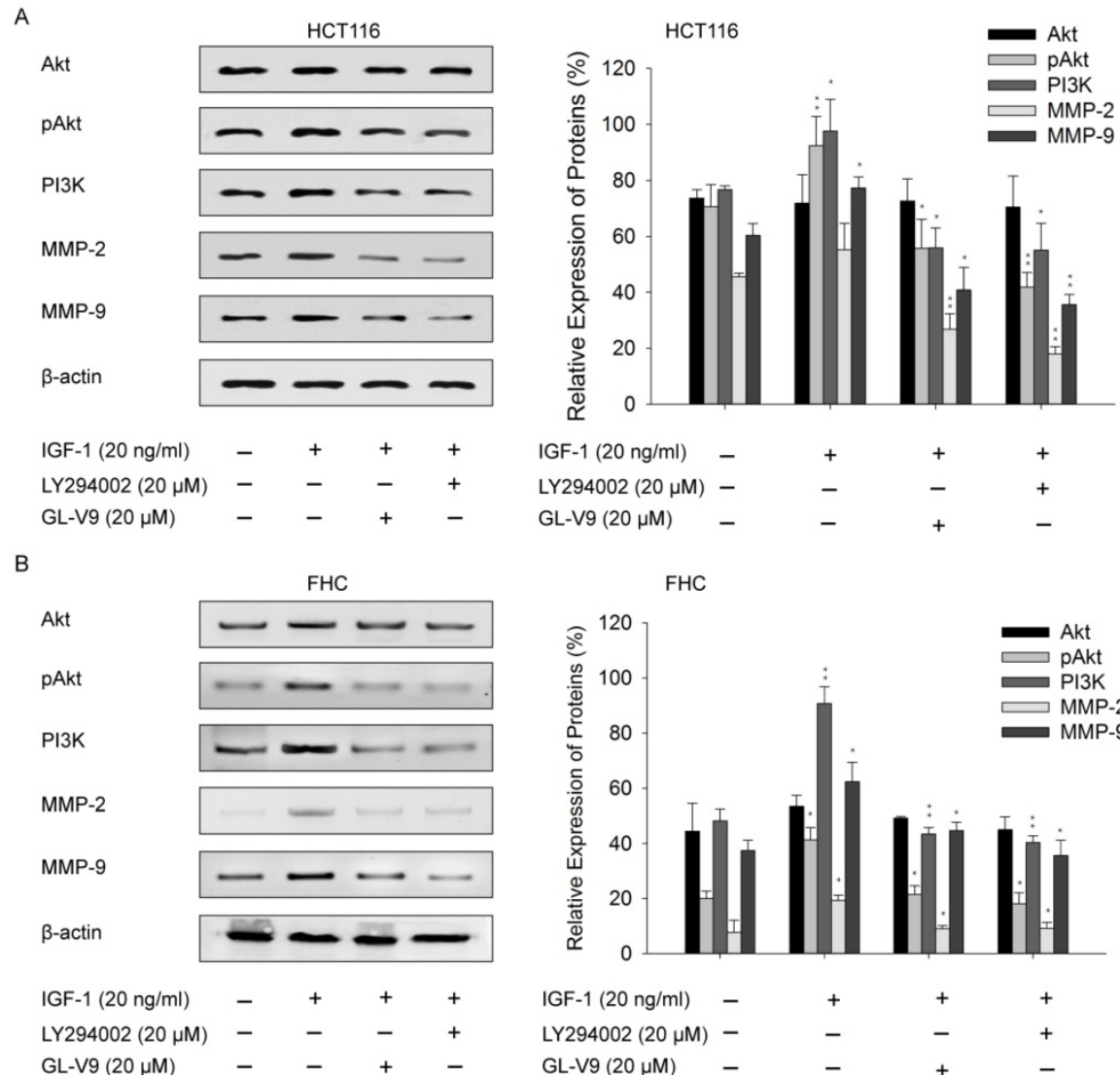

$$
\begin{array}{lllll}
\mathrm{IGF}-1(20 \mathrm{ng} / \mathrm{ml}) & - & + & + & + \\
\mathrm{LY} 294002(20 \mu \mathrm{M}) & - & - & - & + \\
\mathrm{GL}-\mathrm{V} 9(20 \mu \mathrm{M}) & - & - & + & -
\end{array}
$$

GL-V9 $(20 \mu \mathrm{M}) \quad-\quad-\quad+\quad-$
$\mathrm{FHC}$

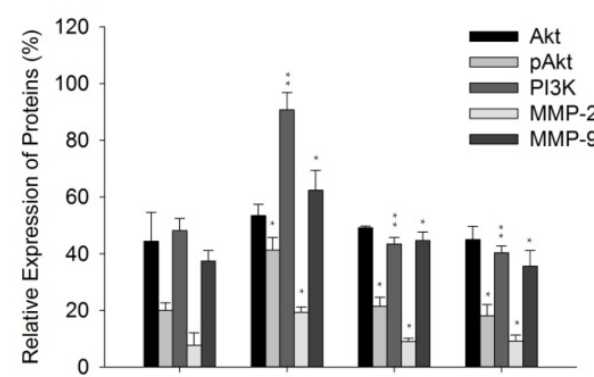

IGF-1 $(20 \mathrm{ng} / \mathrm{ml})$ LY294002 $(20 \mu \mathrm{M})-$ GL-V9 $(20 \mu \mathrm{M})$

Figure 6. Effect of GL-V9 on IGF-1 induced PI3K/Akt activation. (A) CRC cells HCT116 and (B) normal colon epithelial cells FHC were pre-treated with $20 \mu M$ GL-V9 for $24 \mathrm{~h}$ and exposed to $20 \mathrm{ng} / \mathrm{ml}$ IGF- 1 for $2 \mathrm{~h}$. Cells pre-treated with $20 \mu \mathrm{M}$ LY294002 for $24 \mathrm{~h}$ before exposure to IGF-1 were used as positive control. IGF-1 treatment led to increased PI3K expression, enhanced Akt phosphorylation, and up-regulated MMP-2 and MMP-9 expression, while pre-treatment of either GL-V9 or LY294002 significantly reversed IGF-1 induced activation of PI3K/Akt signaling and up-regulation of MMP2 and MMP-9. Relative intensities of western blot bands from three replicated experiments were quantified using the ImageJ software. The expression levels were normalized by $\beta$-actin expression. Statistical significances between the IGF-1 treated group and control group, the IGF-1 treated group and IGF-1 + LY294002/GL-V9 treated groups were calculated. Each error bar represents the mean \pm S.D. of three replicate samples. $* p<0.05$, $* * p<$ 0.01 . 


\section{Discussion}

Wogonin (5,7-dihydroxy-8-methoxyflavone) is a mono-flavonoid isolated from the traditional Chinese medicine Scutellaria radix with various therapeutic potential including anti-inflammatory, anti-oxidative, anticancer activities [21-24]. The high selectivity of wogonin between normal cells and cancer cells makes it a promising agent for cancer treatment [25-27]. As a flavonoid derivative, GL-V9 was synthesized from wogonin through two steps as previous described [16]. In recent years, several studies have demonstrated the beneficial action of GL-V9 on multiple cancer types through regulation of cell apoptosis, cell growth, inflammatory response, and drug resistance $[16,18,19,28,29]$. Apart from its anti-cancer effect, GL-V9 can also ameliorate dextran sulfate sodium (DSS)-induced colonic oxidative stress [30]. Notably, GL-V9 induces the similar biological effects as wogonin at much lower concentrations. In human hepatocellular carcinoma (HCC) cells HepG2, wogonin and GL-V9 effectively induces cell apoptosis at a concentration of $80 \mu \mathrm{M}$ and $20 \mu \mathrm{M}$, respectively [16]. Here we showed that GL-V9 significantly inhibits the invasive and metastatic properties of CRC cells by regulating MMP-2 and MMP-9 expression via PI3K/Akt signaling. To our knowledge, this is the first study to investigate the anti-invasive effect of GL-V9 on CRC cells.

Results from MTT assay showed that GL-V9 inhibited cell viabilities of four CRC cell lines, HCT116, SW480, SW620, and LS174T (Fig. 1). After 24 h-treatment of $20 \mu \mathrm{M}$ GL-V9, the inhibitory rates of the four CRC cell lines varied from $15.6 \%$ to $28.5 \%$, but the inhibitory rate of normal colon epithelial cells was lower than $10 \%$ (Fig. 1). The results indicated the specificity of GL-V9 toward CRC cells. Consistently, a previous study also showed that GL-V9 has little cytotoxic effect on normal bronchial epithelium cells even at a concentration of $80 \mu \mathrm{M}$, suggesting the potential of CL-V9 to target cancer cells without affecting normal tissue [28].

The transwell assay, cell wound healing assay, and cell attachment assay were used to evaluate the effect of GL-V9 on the metastatic potential of CRC and normal colon epithelial cells. The results showed that GL-V9 treatment significantly reduced CRC cell invasion, migration, and adhesion (Fig. 2, 3). Since the malignant degree of normal colon cells are much lower than CRC cells, GL-V9 showed less evident, but still significant inhibitory effect on the migratory, invasion, and adhesive potential of normal cells (Fig. 2 ,3). In agreement with our results, the efficacy of GL-V9 as well as its parent compound wogonin in inhibiting the invasive and migratory capacities of melanoma cells, breast cancer cells and oral cancer cells has been demonstrated in previous studies $[17,31,32]$. In vitro experiments from both our study and previous studies indicated that GL-V9 inhibited cell migration significantly at a concentration $\leq 10 \mu \mathrm{M}$. By contrast, a concentration higher than $30 \mu \mathrm{M}$ is necessary for wogonin to efficiently inhibit cell migration and invasion [17,31]. These evidences implicated that GL-V9 may be a more potent candidate for prevention of tumor metastasis.

Tumor distant metastasis, which occurs in approximately $20 \%$ of patients at the time of initial diagnosis, is the primary cause of death in CRC [33]. Elucidation of the molecular mechanism underlying CRC metastasis and identification of novel therapeutic targets have been the research hotspots in the past few years. It is well known that local invasion is the first requirement for distant metastasis, while a key event in tumor invasion is degradation of the surrounding ECM, which involves activation of MMP-2 and MMP-9 [6,8]. In CRC patients, expression of MMP-2 and MMP-9 are significantly correlated with disease stage, long-term survival, and clinical outcome [34-37].

The MAPK/ERK and PI3K/Akt signaling pathways are pivotal for many fundamental cellular processes such as cell proliferation, invasion, and differentiation [38,39]. It is documented that induction of MAPK/ERK or PI3K/Akt contributes to cancer cell invasion by regulating MMP-2 and/or MMP-9 [40-42]. In this study, we found that GL-V9 treatment reduced the expression and activities of MMP-2 and MMP-9 in CRC cells (Fig. 4). Meanwhile, expression of PI3K and the phosphorylated form of Akt was significantly decreased (Fig. 5). On the contrary, GL-V9 did not affect the expression of MAPK p38 and both the total and phosphorylated forms of ERK1/2 (Fig. 5), indicating that GL-V9 probably inhibits CRC cell invasion and MMPs expression mainly through PI3K/Akt signaling. Since involvement of the PI3K/Akt/MMP-2/9 axis in CRC metastasis has been reported before $[43,44]$, we further investigate the effects of combined treatment using GL-V9 and Akt activator IGF-1 on the expression of MMP-2 and MMP-9. Our results confirmed that for both CRC cells and normal colon epithelial cells, activation of Akt signaling led to increased MMP-2 and MMP-9 expression, while treatment of GL-V9 or the specific Akt inhibitor LY294002 counterbalanced the effect caused by IGF-1 (Fig. 6). However, given that some other modulators of the MMPs activity, such as the hedgehog signaling and NF-kB signaling pathways have also been identified [44,45], whether the inhibitory effects of GL-V9 on MMP-2 and MMP-9 expression are solely dependent on PI3K/Akt signaling remain to be further confirmed. 
In conclusion, our study demonstrated that GL-V9 could inhibit the invasion and metastasis of CRC cells in vitro. The inner molecular mechanisms involved inhibition of GL-V9 on the expression and activities of MMP-2 and MMP-9 possibly via suppression of the PI3K/Akt signaling. Taken together with previous studies showing its anti-tumor effect, GL-V9 appears to be a promising therapeutic agent of CRC.

\section{Acknowledgements}

This project was supported by Hangzhou Peak Discipline of Gastroenterology, the Key Laboratory of Integrated Traditional Chinese and Western Medicine for Biliary and Pancreatic Diseases of Zhejiang Province, the Key Laboratory of Clinical Cancer Pharmacology and Toxicology Research of Zhejiang Province (2020E10021), the Science and Technology Project of Hangzhou Health Commission (A20200113), the Zhejiang Medical and Health Science and Technology Plan (Grant No. WKJ-ZJ-2136 2019RC068 and 2021437779), and the Hangzhou Medical and Health Science and Technology Plan (Grant No. 2016ZD01, OO20190610 and A20200174). The funders had no role in study design, data collection and interpretation, or the decision to submit the work for publication.

\section{Competing Interests}

The authors have declared that no competing interest exists.

\section{References}

1. Ferlay J, Colombet M, Soerjomataram I, et al. Estimating the global cancer incidence and mortality in 2018: GLOBOCAN sources and methods. Int J Cancer. 2019; 4(8): 1941-1953.

2. Freddie B, Jacques F, Isabelle S, et al. Global cancer statistics 2018: GLOBOCAN estimates of incidence and mortality worldwide for 36 cancers in 185 countries. CA-Cancer J Clin. 2018; 68(6): 394-424.

3. Aimery DG. Adjuvant Therapy of Stage II and III Colon Cancer. Semin Oncol. 2005; 32(6):11-14.

4. Yilmaz M, Christofori G, Lehembre F. Distinct mechanisms of tumor invasion and metastasis. Trends Mol. Med. 2007; 13(12): 535-541.

5. Chaudhary AK, Pandya S, Ghosh K, et al. Matrix metalloproteinase and its drug targets therapy in solid and hematological malignancies: an overview. Mutat Res. 2013; 753: 7-23.

6. Vihinen $\mathrm{P}$, Ala-aho $\mathrm{R}$, Kahari VM, et al. Matrix metalloproteinases as therapeutic targets in cancer. Curr Cancer Drug Tar. 2005; 5: 203-220.

7. Freije JM, Balbin M, Pendas AM, et al. Matrix metalloproteinases and tumor progression. Adv Exp Med Biol. 2003; 532:91-107.

8. Hanemaaijer R. Increased gelatinase-A and gelatinase-B activities in malignant vs. benign breast tumors. Int J Cancer. 2000; 86: 204-207.

9. Zeng ZS, Cohen AM, Guillem JG, et al. Loss of basement membrane type IV collagen is associated with increased expression of metalloproteinases 2 and 9 (MMP-2 and MMP-9) during human colorectal tumorigenesis. Carcinogenesis. 1999; 20(5): 749-755.

10. Arajo RF, Lira GA, Vilaa JA, et al. Prognostic and diagnostic implications of MMP-2, MMP-9, and VEGF-a expressions in colorectal cancer. Pathol Res Pract. 2015; 211(1):71-77.

11. Salem N, Kamal I, Al-Maghrabi J, et al. High expression of matrix metalloproteinases: MMP-2 and MMP-9 predicts poor survival outcome in colorectal carcinoma. Future Oncol. 2016; 12(3): 323-331.

12. Li JP, Teng YH, Liu SL, et al. Cinnamaldehyde affects the biological behavior of human colorectal cancer cells and induces apoptosis via inhibition of the PI3K/Akt signaling pathway. Oncol Rep. 2015; 35(3): 1501-1510.
13. Chen SQ, Chen W, Zhang X, et al. Overexpression of KiSS-1 reduces colorectal cancer cell invasion by downregulating MMP-9 via blocking PI3K/Akt/NFkappa B signal pathway. Int J Oncol, 2016; 48: 1391-1398.

14. Hsu HH, Liu CJ, Shen CY, et al. p38a MAPK mediates 17ß-estradiol inhibition of MMP-2 and -9 expression and cell migration in human lovo colon cancer cells. J Cell Physiol. 2012; 227(11): 3648-3660.

15. Li S, Ung TT, Nguyen TT, et al. Cholic Acid Stimulates MMP-9 in Human Colon Cancer Cells via Activation of MAPK, AP-1, and NF-KB Activity. Int J Mol Sci. 2020; 21(10): 420

16. Li LW, Lu N, Dai QS, et al. A newly synthetic flavonoid derivative, induces mitochondrial-mediated apoptosis and G2/M cell cycle arrest in human hepatocellular carcinoma HepG2 cells. Mol Cell Pharmacol, 2011; 670(1): 13-21.

17. Li LW, Chen P, Ling Y, et al. Inhibitory effects of GL-V9 on the invasion of human breast carcinoma cells by downregulating the expression and activity of matrix metalloproteinase-2/9. Eur J Pharm Sci. 2011; 43: 393-399

18. Zhao Y, Guo QL, Zhao K, et al. Small molecule GL-V9 protects against colitis-associated colorectal cancer by limiting NLRP3 inflammasome through autophagy. Oncoimmunology. 2018; 7(1): e1375640.

19. Zhao K, Li GJ, Yao YY, et al. Activation of phospholipase $\mathrm{C}-\gamma 1$ and translocation of phosphatidylinositol-3,4,5-trisphosphate 3-phosphatase contribute to GL-V9-induced apoptosis in human gastric cancer cells. Exp Cell Res. 2017; 356(1): 8-19.

20. Yan L, Borregaard N, Kjeldsen L, et al. The high molecular weight urinary matrix metalloproteinase (MMP) activity is a complex of gelatinase B/MMP-9 and neutrophil gelatinase-associated lipocalin (NGAL). Modulation of MMP-9 activity by NGAL. J Biol Chem. 2001; 276(40), 37258-37265.

21. Chen LG, Huang LY, Tsai KW, et al. Wogonin, a bioactive flavonoid in herbal tea, inhibits inflammatory cyclooxygenase- 2 gene expression in human lung epithelial cancer cells. Mol Nutr Food Res. 2008; 52: 1349-1357.

22. Lee $\mathrm{H}$, Kim $\mathrm{YO}$, Kim $\mathrm{H}$, et al. Flavonoid wogonin from medicinal herb is neuroprotective by inhibitinginflammatory activation of microglia. FASEB J. 2003; 17: 1943-1944.

23. Lee YM, Cheng PY, Chen SY, et al. Wogonin suppressesarrhythmias, inflammatory responses, and apoptosis induced by myocardialischemia/ reperfusion in rats. J Cardiovasc Pharmacol. 2011; 58: 133-142.

24. Piao HZ, Jin SA, Chun HS, et al. Neuroprotective effectof wogonin: potential roles of inflammatory cytokines. Arch Pharm Res. 2004; 27: 930-936.

25. Himeji M, Ohtsuki T, Fukazawa H, et al. Difference of growth-inhibitoryeffect of Scutellaria baicalensis-producing flavonoid wogonin among humancancer cells and normal diploid cell. Cancer Lett. 2007; 245: 269-274.

26. Wei $\mathrm{L}, \mathrm{Lu} \mathrm{N}, \mathrm{Dai} \mathrm{Q}$, et al. Different apoptoticeffects of wogonin via induction of $\mathrm{H}(2) \mathrm{O}(2)$ generation and $\mathrm{Ca}(2+)$ overload inmalignant hepatoma and normal hepatic cells. J Cell Biochem. 2010; 111: 1629-1641.

27. Polier G, Ding J, Konkimalla BV, et al. Wogonin and related nat-ural flavones are inhibitors of CDK9 that induce apoptosis in cancer cells bytranscriptional suppression of Mcl-1. Cell Death Dis. 2011; 2: e182.

28. Zhang $X$, Kang $Y$, Huo $T$, et al. GL-V9 induced upregulation and mitochondrial localization of NAG-1 associates with ROS generation and cell death in hepatocellular carcinoma cells. Free Radic Biol Med. 2017; 112:49-59.

29. Zhao L, Li W, Zhou $\mathrm{Y}$ et al. The overexpression and nuclear translocation of Trx-1 during hypoxia confers HepG2 cells resistance to DDP and GL-V9 reverses the re sistance by suppressing Trx-1/ref-1 axis. Free Radic Biol Med. 2015; 82: 29-41.

30. Zhao $Y$, Sun $Y$, Ding $Y$, et al. GL-V9, a new synthetic flavonoid derivative, ameliorates DSS-induced colitis against oxidative stress by up-regulating Trx-1 expression via activation of AMPK/FOXO3a pathway. Oncotarget. 2015; 6(28): 26291-307.

31. Zhao $\mathrm{K}$, Wei LB, Hui $\mathrm{H}$, et al. Wogonin supresses melanoma cell B16-F10 invasion and migration by inhibiting Ras-Medicated pathways. Plos One. 2014; 9(9): e106458

32. Ho YC, Lee SS, Yang SF, et al. Inhibitory effects of wogonin on invasion by human oral cancer cells by decreasing the activity of matrix metalloproteinases and urokinase-plasminogen activator - ScienceDirect. J Dent Sci. 2014; 9(2):172-7.

33. Kopetz S, Chang GJ, Overman MJ, et al. Improved survival in metastatic colorectal cancer is associated with adoption of hepatic resection and improved chemotherapy. J Clin Oncol. 2009; 27(22): 3677-83.

34. Waas ET, Hendriks $\mathrm{T}$, Lomme $\mathrm{RM}$, et al Plasma levels of matrix metalloproteinase 2 and tissue inhibitor of metalloproteinase 1 correlate with disease stage and survival in colorectal cancer patients. Dis Colon Rectum. 2005; 48: 700710 .

35. Kim EY, Song HY, Kim JC, et al. Mmp9 expression after metallic stent placement in patients with colorectal cancer: association with instent restenosis. Radiology. 2014; 271: 901908.

36. Koskensalo S, Hagström J, Linder N, et al. Lack of MMP9 expression is a marker for poor prognosis in Dukes' B colorectal cancer. BMC Clin Pathol.2012; 12: 24

37. Langers AM, Verspaget HW, Hawinkels LJ, et al. MMP2 and MMP9 in normal mucosa are independently associated with outcome of colorectal cancer patients. Br J Cancer. 2012; 106: 14951498.

38. Wen X, Zhu J, Dong L, et al. The role of c2orf68 and PI3K/Akt/mTOR pathway in human colorectal cancer. Med Oncol. 2014; 31(8): 92. 
39. Sainz RM, Hevia D, Quiros I, et al. MAPK/ERK signaling mediates melatonininduced neuroendocrine differentiation in prostate cancer cells. Ejc Supplements.2008; 6(9):86.

40. Kajanne R, Miettinen P, Mehlem A, et al. EGF-R regulates MMP function in fibroblasts through MAPK and AP-1 pathways. J. Cell Physiol. 2007; 212: 489-97.

41. Chen JS, Huang $\mathrm{XH}$, Wang $\mathrm{Q}$, et al. Sonic hedgehog signaling pathway induces cell migration and invasion through focal adhesion kinase/AKT signaling mediated activation of matrix metalloproteinase (MMP)2 signaling pathway. J Cell Biochem. 2008; 104: 1526.

42. Chen JS, Wang $\mathrm{Q}, \mathrm{Fu} \mathrm{XH}$, et al. Involvement of PI3K/PTEN/AKT/mTOR pathway in invasion and metastasis in hepatocellular carcinoma : Association with MMP-9. Hepatol Res. 2010; 39(2):177-86.

43. Rui $X$, Yan $X$, Zhang $K$. Baicalein inhibits the migration and invasion of colorectal cancer cells via suppression of the AKT signaling pathway. Oncol Lett. 2016; 11(1): 685-8.

44. Liu JY, Wu XY, Wu GN, et al. FOXQ1 promotes cancer metastasis by PI3K/AKT signaling regulation in colorectal carcinoma. Am J Transl Res. 2017; 9(5):2207.

45. Fan H, Li H, Liu G, et al. Doxorubicin combined with low intensity ultrasound suppresses the growth of oral squamous cell carcinoma in culture and in xenografts. J Exp Clin Canc Res. 2017; 36(1): 163.

46. Tsai JR, Liu PL, Chen YH, et al. Curcumin Inhibits Non-Small Cell Lung Cancer Cells Metastasis through the Adiponectin/NF-kb/MMPs Signaling Pathway. Plos One. 2015; 10(12): e0144462. 